THE VERULAM REVIEW AND THE BRITISH MEDICAL ASSOCIATION.

THE British Medical Association is to be congratulated on the result of the action for libel brought against it by the editor of the Verulam Review. Although we entirely fail to see how any one who had written such letters as were shown in the trial to have been written by the plaintiff could ever have persuaded himself that he had any proper cause of complaint against the British Medical Journal, for the very moderate comment on his proceedings which had appeared in that journal, juries are proverbially skittish in libel cases, and there always was the chance that some prejudice might be imported into the case by its connection with the vivisection question in regard to which the dispute arose. It is very satisfactory, then, to find that the verdict was for the defendant, with costs, It is not, however, in its relation either to the plaintiff or the defendant that the interest of this trial centres so far as the outside world is concerned, but rather in the fact that opportunity was given in its course for witnesses to state on oath certain facts in regard to which much ignorance and misconception exist among some, and as to which erroneous assertions are often made by others. Dr. Vivian Poore repeated on oath the statement made in his Report for 1897, that " the only experiments performed without anæsthetics are of the nature of inoculations or hypodermic injections." A great deal of the evidence centred round the so-called "Certificate A," under which operations can be performed without anæsthetics, and it was brought out in evidence with the greatest clearness that this certificate must bear the nature of the operation for which it is granted on the face of it, and that these certificates never have been used for "serious operations other than inoculation.". Professor Sims Woodhead, who has been for a long time at the head of a large laboratory, said that "leaving out inoculation experiments he had not seen in his laboratory an experiment upon a living cat or dog without an anæsthetic"; and, further, that " he had no experience of any painful experiments upon animals in this country without anæsthetics." People are so constantly being told of the "horrors" that are going on in regard to experiments upon animals that they find it difficult to believe that most of what they hear is all moonshine. Perhaps, now that the above statements have been made on oath in a Court of Justice, they will be more satisfied.

\section{THE TREATMENT OF LUPUS BY X-RAYS.}

IN view of the active, though injurious effects, which have from time to time been observed to result from the use of the $x$ rays, and of the fact that these effects wondered extend far below the surface, it is not to be endeavod at that many investigators have been led to of use in to bring these powerful rays into some sort fessed that in treatment of disease. It must be con. exact relation our present state of ignorance as to the tions of radiant energy the $x$ rays and other manifesta. speculatediant energy it is almost waste of time to Even in as to the mode in which the $x$ rays do good. heat, or by radiant light as lately practised by Finsen, we can but guess as to the manner in which the curative effects are produced. What we do know about these proceedings seems to be that, whether it is a question of light, or heat, or $x$ rays, or, maybe, of some form of electrical vibration emanating from the radiant points, the different tissues offer varying degrees of resistance to their transmission, and it is but reasonable to believe that where such resistance occurs it is accompanied by some tissue change, some phagocytosis, perhaps-temporary it may be, but sufficient to exert some influence upon morbid or lowly organised tissues, besides the directly germicidal influence which $x$ rays, as well as direct light rays, may exert upon pathogenic microbes. Be this as it may, the evidence seems pretty clear that a very marked improvement is produced in cases of lupus by exposing the diseased patch to the full effect of the $x$ rays. Dr. Eduard Schiff, of Vienna, claims to have been the first to cure lupus in this way, but since then the method has been tried in a fair number of cases. A remarkably good example of the successful use of the rays for this purpose is reported by Dr. R. E. Scholefield, in the British Medical Journal for May 5th. The nose was the part affected, and in using the rays the rest of the face was carefully protected. From the photographs given no one can doubt the beneficial effect of the applications.

\section{THE RED CROSS.}

IN a letter to a contemporary, ${ }^{1}$ Sir William Thomson says that the present arrangements for displaying the Red Cross flag are quite insufficient to ensure that the nature of the tent to which it is attached shall be made clear to the enemy; and he considers that the practical invisibility of the flag is accountable for some of the cases in which the Boers have unwittingly, as he suggests, fired upon ambulances and hospitals, especially the latter. He says that under certain circumstances the Geneva Red Cross, displayed as it usually is upon a flag, is practically invisible, and he gives an instance. "The morning was hot and sultry. Thẹre was hardly a sign of motion in the air. I said to a distinguished officer, 'If this camp were attacked by the enemy now, how could they identify your hospital?' ' Oh,' he said, 'we have our Red Cross flags up.' I asked, 'Where?' He answered, 'There, and there, and there.' 'Oh, yes,'I said, 'they are up, but who could see them at a thousand yards off?' And I pointed out that the flags were lying motionless along the flagstaff in folds." He adds that he can quite understand how an enemy could send shell after shell into a hospital under the belief that he was attacking a combatant portion of the army, for an enemy might see only a group of tents precisely similar to those used by the fighting men, and if the wind were not sufficient to extend the flag there would be nothing to tell him that he was firing on a crowd of sick and wounded men. At the distance at which shots are now fired even the most careful search with a telescope would not enable anyone to identify the hospital. Sir William suggests that instead of flags being hung from a pole they should be stretched upon a frame of some sort, but at any rate that some better method than that at present in use should be employed for indicating the hospital buildings.

I Brit. Med. Jour., May 5. 